\title{
ILCEA
}

Revue de l'Institut des langues et cultures

d'Europe, Amérique, Afrique, Asie et Australie

$11 \mid 2009$

Langues \& cultures de spécialité à l'épreuve des

médias

\section{Journaliste et traducteur : deux métiers, deux réalités}

\section{Élisabeth Lavault-Olléon et Véronique Sauron}

\section{OpenEdition}

Journals

Édition électronique

URL : http://journals.openedition.org/ilcea/210

DOI : 10.4000/ilcea.210

ISSN : 2101-0609

Éditeur

UGA Éditions/Université Grenoble Alpes

Édition imprimée

ISBN : 978-2-84310-179-3

ISSN : 1639-6073

Référence électronique

Élisabeth Lavault-Olléon et Véronique Sauron, « Journaliste et traducteur : deux métiers, deux réalités », ILCEA [En ligne], 11 | 2009, mis en ligne le 30 avril 2009, consulté le 08 mars 2021. URL: http://journals.openedition.org/ilcea/210 ; DOI : https://doi.org/10.4000/ilcea.210

Ce document a été généré automatiquement le 8 mars 2021

(C) ILCEA 


\title{
Journaliste et traducteur : deux métiers, deux réalités ${ }^{1}$
}

\author{
Élisabeth Lavault-Olléon et Véronique Sauron
}

Dans le vaste domaine de la communication, journalistes et traducteurs ont en commun d'être des professionnels qui produisent du discours, les premiers à partir d'événements, les seconds à partir de textes. Dans le cadre de la traduction spécialisée ou pragmatique qui est le nôtre, si le traducteur part des textes, c'est avant tout pour en transmettre le sens, défini dans sa contextualité (Seleskovitch et Lederer, 1984, Israël et Lederer, 2005), et dans sa fonctionnalité, toujours intégrée dans une situation de communication spécifique (Vermeer, 1996). Quant au journaliste, l'événement qu'il rapporte et commente est souvent déjà l'objet d'autres textes sur lesquels il s'appuie, même lorsque ceux-ci sont écrits dans une autre langue que la sienne. Contextualité et intertextualité sont deux éléments clés de leur pratique d'écriture commune. La défense d'une langue précise, claire et riche en nuances est un autre argument traditionnel qui les rapproche: "plusieurs métiers, mais une seule langue, un seul combat » soulignait récemment un correcteur au journal Le Monde (Colignon, 2007 : 50). Leurs activités se rapprochent-elles au point de coexister ou de se substituer l'une à l'autre? Le sujet mériterait de longs développements et nous n'en explorerons cidessous que quelques aspects qui touchent à notre activité de traductrices.

\section{Le traducteur et la presse}

\section{Des traducteurs journalistes}

2 Il existe des traducteurs qui sont aussi des journalistes, particulièrement dans les pays où la recherche et la communication d'informations ne peuvent exister que grâce à une activité bilingue ou plurilingue, comme au Moyen-Orient par exemple. En France, pays très majoritairement monolingue, les deux métiers sont généralement différenciés. Néanmoins, la traduction est souvent considérée comme une école d'écriture, qui a permis à de nombreux hommes et femmes de plume, plutôt auteurs de fiction il est 
vrai, de faire leurs armes. Certains, comme Guillaume Apollinaire, ont effectivement associé le journalisme et la traduction à leur pratique d'écriture littéraire.

3 C'est manifestement lorsqu'il traduit la presse que le traducteur se rapproche le plus $\mathrm{du}$ journaliste. De même qu'un traducteur littéraire a forcément en lui un écrivain latent, un traducteur de presse générale ou spécialisée prend plaisir à adopter la qualité d'un style journalistique correspondant au média et au public pour lesquels il traduit. À lui de recréer un titre et un chapeau accrocheurs, d'expliciter un sigle ou une réalité culturelle inédite, en bref d'adapter son texte à son lecteur, comme l'a fait le journaliste lorsqu'il a écrit le texte original, dans une démarche proprement cibliste (Ladmiral, 1994). C'est pour cette raison, entre autres, que la traduction journalistique, aussi qualifiée d'éditoriale, connaît dans les universités un tel succès et s'impose comme un genre de traduction modèle.

\section{Traduction journalistique à l'université}

4 La pratique de la traduction à l'université fait la part belle à la presse, en tout cas dans les filières de langues étrangères appliquées. Lorsque les formations universitaires en langues étrangères se sont ouvertes au monde économique par la création de ces filières, les universitaires y ont remplacé la classique version littéraire par la traduction d'extraits d'articles de presse, en choisissant de préférence la presse de niveau soutenu, l'exemple type en anglais étant l'hebdomadaire britannique The Economist ${ }^{2}$. Ces articles permettent en effet un intéressant travail d'entraînement à la traduction puisqu'ils cumulent des difficultés linguistiques, à la fois de compréhension et de transfert (lexique et syntaxe soutenus, style particulier de chaque média), tout en nécessitant de la part de l'étudiant traducteur des connaissances mises à jour sur l'actualité. Le repérage des références ou allusions et des réalités culturelles spécifiques décrites dans ces articles doit s'accompagner d'une stratégie de traduction appropriée, par l'adaptation, l'explicitation ou la neutralisation, par exemple. Il existe un très grand nombre de manuels qui présentent et commentent des traductions d'articles de presse ${ }^{3}$. Si cette richesse explique le succès de ces cours de traduction, elle a aussi entretenu l'idée fausse que ce type de texte reflétait l'activité des traducteurs professionnels, spécialisés ou non. Or il n'en est rien. Sur le marché de la traduction, les besoins en traduction journalistique sont inférieurs à la représentation qui en est faite à l'université, et bien inférieurs à la demande pour d'autres documents de types très divers, tels que les contrats, rapports, brochures, logiciels, modes d'emploi, jeux, notes techniques, nomenclatures, etc. (Gouadec, $2002: 8$ ).

\section{Besoins en traduction de la presse}

Dans quels cas fait-on appel à des traducteurs professionnels pour traduire des articles de presse, sur le marché français? L'hebdomadaire Courrier international, qui vient aussitôt à l'esprit, reste un cas marginal (moins d'une quinzaine de traducteurs sur place). Quelques grandes revues diffusent des versions traduites, comme le National Geographic Magazine, traduit désormais en 32 langues depuis sa première édition japonaise de 1995. Le cas de Pour la science, édition française du Scientific American mériterait une étude à lui seul car les articles sont généralement réécrits par des journalistes scientifiques à partir des traductions. Malgré ces exemples, un petit sondage informel auprès de traducteurs de statuts variés donne les tendances 
suivantes : articles de presse générale : de 0 à $1 \%$ de l'activité de traduction; articles de presse spécialisée entre 0 et $5 \%$, sauf certains traducteurs très spécialisés qui peuvent traduire jusqu'à $50 \%$ pour des revues techniques. On constate néanmoins une augmentation récente de la demande, due à l'expansion des grandes chaînes audiovisuelles d'information internationale qui diffusent en deux langues (comme France 24 ou Al-Jazira) ou plus (sept langues pour Euronews) et entretiennent un site Internet bilingue ou multilingue. Parallèlement, les grands titres de la presse écrite ont aussi leur portail Internet, qui peut proposer, entre autres, des articles traduits de la presse étrangère : par exemple, les articles de Newsweek traduits sur le site du Nouvel Observateur dans le cadre de la campagne présidentielle américaine $2008^{4}$. Autre exemple, une société de traduction s'est récemment vu proposer un contrat portant sur la traduction quotidienne d'une revue de presse, d'allemand en français, représentant un volume d'environ 2500 à 3000 mots à traduire entre 9 heures 30 et 13 heures pour une mise en ligne dans l'après-midi 5 .

On notera enfin l'apparition de nouveaux métiers associant la recherche d'information et la traduction, principalement dans des contextes de veille, technologique ou commerciale par exemple, ou encore dans "l'analyse médias", dont les experts «mènent des recherches qualitatives et quantitatives dans deux ou trois langues pour répondre à une demande d'information précise, régulière ou ponctuelle " (Franjié, $2007: 63)$ pour un service de communication ou une agence de publicité internationale. De même, "l'infomédiation ", qui associe la traduction et la synthèse, effectuées par des «lecteurs-rédacteurs-traducteurs» est une "nouvelle activité spécialisée qui consiste à rechercher et à collecter l'information sur le plan international dans tous les médias, à concevoir des panoramas de presse et/ou des synthèses de ces panoramas et à les diffuser auprès d'une clientèle » (Lebtahi et Ibert, $2004: 225)$.

7 Parmi les pratiques de traduction journalistique plus classiques, les communiqués de presse sont le pain quotidien des traducteurs, bien plus que les articles. Ce sont des textes d'information transmis à la presse pour publication partielle et gratuite. Toujours courts (en moyenne une page), ils contiennent une information précise (annonce d'un événement, sortie d'un nouveau produit, changements dans une organisation), qui est destinée à être diffusée dans les médias. Rédigés par le service marketing ou communication des entreprises et organisations, plus rarement par des attachés de presse, ils répondent à des exigences très strictes en termes de format et de style. L'image et la promotion des organisations dépendent de la réception de leurs communiqués par la presse générale et spécialisée puisque ce sont les journalistes qui en reproduisent une partie dans leurs articles. C'est pourquoi la traduction en est généralement confiée à des traducteurs professionnels. La traduction des communiqués de presse est un secteur où traducteurs et journalistes sont en contact étroit : selon René Meertens ${ }^{6}$, « comme un journaliste est toujours pressé, les informations doivent être immédiatement exploitables, "précuites", pour qu'il puisse lui-même les communiquer à ses lecteurs en procédant à un minimum de réécriture. En conséquence, l'auteur [le traducteur] du communiqué doit respecter toutes les règles qui s'imposent au journaliste ». Résultat de cette chaîne de transmission qui part du rédacteur pour parvenir au grand public par le truchement du traducteur puis du journaliste: un traducteur de communiqués d'organisations internationales, par exemple, peut entendre ses propres traductions de la journée reprises aux informations du journal du soir. 
Cela signifie aussi que, la plupart du temps, les journalistes n'ont pas à assurer de traduction puisqu'il se fondent sur des communiqués de presse déjà traduits, ou sur des dépêches d'agence qui sont, elles aussi, souvent traduites. C'est le cas à l'Agence France Presse dont "les services généraux [...] proposent aux professionnels des médias et hors médias, 100000 mots à 300000 mots par jour bien maîtrisés, en six langues (français, anglais, espagnol, portugais, allemand et arabe) et en continu $»^{7}$.

\section{Le journaliste et la traduction}

\section{Des journalistes traducteurs}

9 Même si les journalistes peuvent s'appuyer sur des dépêches et communiqués traduits par des professionnels, la rapidité de l'information les oblige souvent à chercher leurs sources dans des articles et communiqués en version originale qu'ils traduisent euxmêmes, plus ou moins bien, et souvent trop vite : il se substituent alors au traducteur.

Ce phénomène récent s'inscrit dans la mondialisation des échanges : information quasi immédiate sur les médias audiovisuels, souvent traduite dans l'urgence, puis reprise par les médias écrits. L'information permanente en ligne permet en outre aux journalistes de s'appuyer sur des articles étrangers non traduits, sans qu'ils songent toujours à faire les vérifications linguistiques nécessaires. Il en résulte des formulations qui peuvent surprendre certains lecteurs avertis qui expriment régulièrement des critiques dans les forums de commentaires affichés à la suite des articles disponibles sur Internet. Les traducteurs professionnels ne sont pas en reste et déplorent régulièrement des maladresses ou des inexactitudes dans les propos rapportés par les journalistes à partir de sources étrangères ${ }^{8}$.

11 Les exemples qui suivent montrent comment certains événements ont été relayés dans la presse par des journalistes sur la base d'informations publiées en langue étrangère qui n'ont pas été appréhendées dans toute leur dimension linguistique, sémiotique et culturelle, ou qui ont été interprétées, modifiant la perception que peut en avoir le lecteur cible ${ }^{9}$.

\section{Études de cas}

12 Un premier exemple concerne un épisode politique qui a alimenté la presse au mois de novembre 2007. Lors du sommet ibéro-américain qui s'est déroulé à Santiago du Chili, une altercation a opposé le président vénézuelien Hugo Chavez au roi d'Espagne Juan Carlos. Alors que le premier ministre espagnol Jose Zapatero intervient à la tribune en demandant à Hugo Chavez, qui vient de qualifier Jose Maria Aznar de fasciste, de faire preuve de respect, le président vénézuélien ne cesse de parler, empêchant le représentant espagnol de s'exprimer. Irrité, le roi d'Espagne s'avance sur son siège, lui tend une main ouverte en lui demandant en espagnol : « ¿Por qué no te callas? ».

13 L'incident a été largement commenté dans nombre de quotidiens en Espagne et dans le monde entier. Les internautes français ont pu consulter, sur le site Internet du Nouvel Observateur, une vidéo accompagnée du commentaire suivant :

« La dernière journée des travaux du Sommet libéro-latino américain de Santiago a connu un incident quand le roi d'Espagne, excédé par les interruptions de parole du président vénézuélien a demandé à Hugo Chavez de « la fermer ». « Pourquoi ne la 
bouclez-vous pas?», a lancé Juan Carlos en montrant du doigt Chavez qui tentait

d'interrompre le chef du gouvernement espagnol José Zapatero. » roman à succès était traduite consécutivement par le journaliste lui-même. Il y était question du mode de vie très libre des étudiants américains, particulièrement favorisé par "les dortoirs mixtes des campus américains", ce qui pouvait sembler étonnant dans le contexte. Il s'agissait en fait des dorms, abréviation de dormitories qui, aux ÉtatsUnis, désignent, non pas des dortoirs comme en Grande-Bretagne, mais des résidences universitaires. La personne qui a traduit, peut-être par nécessité et prise par le temps, ne connaissait pas la différence de sens entre l'anglais britannique et américain ni la réalité des campus américains.

18 Autre exemple, autre cas : durant la crise boursière, on a beaucoup parlé de marché des futures, expression incompréhensible pour les novices en matière financière, mais pas 
pour les financiers qui connaissent le terme anglais futures market. Le problème, c'est que la réalité du marché à terme ou du marché de contrats à terme existe depuis longtemps, avec une appellation française précise qui ne nécessite en aucun cas un emprunt à l'anglais. Et surtout pas un calque qui, non seulement ne comble pas une lacune terminologique, mais introduit des malentendus évidents ${ }^{12}$. D'après l'office de la langue française du Québec:

"Les termes marché des futurs et marché des futures sont employés sous l'influence de l'anglais futures market et sont à éviter, car, en français, le terme futur n'a pas le sens de 'contrat à terme' et le substantif future est inexistant. Les emprunts futur et future sont inappropriés pour dénommer une transaction boursière par laquelle une prise ferme de l'actif est prévue à un terme donné (date ultérieure à la négociation), convenu entre les contractants. $»^{13}$

19 En France, le Journal officiel du 22 septembre 2000 recommande le terme marché de contrats à terme ${ }^{14}$.

20 La question des subprimes, qui a envahi nos ondes et nos écrans à l'automne 2007 est plus délicate. Il s'agit là d'une réalité financière américaine qui n'avait pas encore d'équivalent officiel en français, désignant des crédits hypothécaires à taux variable accordés à des personnes peu solvables qui ne peuvent bénéficier de taux plus avantageux. On voit la difficulté de rendre compte de cette réalité en français en essayant de "faire court », subprime étant un raccourci de subprime loan (prêt à haut risque) ou subprime mortgage (prêt hypothécaire à haut risque) ou subprime product (produit à risques). Le tableau ci-dessous récapitule diverses solutions adoptées.

Elle a aussi annoncé 600 millions de pertes supplémentaires liées aux « subprime ». ${ }^{15}$

La banque suisse UBS annonce ce lundi avoir enregistré d'importantes pertes au troisième trimestre liées à la crise du subprime. ${ }^{16}$

Cette nouvelle crise financière pourrait durer deux ans, bien davantage que celle des crédits hypothécaires " subprime ${ }^{17}{ }^{17}$

Pourtant, cette année, la plus grande humilité s'impose en matière de pronostics. L'économie américaine atterrit, et le "subprime crédit " continuera de générer des turbulences en 2008 comme il l'a fait depuis un semestre. ${ }^{18}$

Il souligne que les perturbations financières nées aux États-Unis en raison de la crise des crédits hypothécaires à risques «subprime » ont aussi touché l'Europe occidentale et que la croissance dans ces deux zones économiques va fortement ralentir, voire même caler temporairement aux États-Unis. ${ }^{19}$

La crise financière née cet été s'éternise. Provoquée par la faillite d'un segment de marché a priori circonscrit et localisé, celui du crédit hypothécaire à risque (subprime mortgage) aux États-Unis, elle menace la stabilité financière de la planète. ${ }^{20}$

Jusqu'ici, les banquiers se pensaient, au moins en partie, « assurés » contre les pertes dues à la crise des crédits immobiliers américains à risque, dits "subprime ». Près de 800 milliards de dollars de crédits structurés contenant des subprime (en jargon technique : des CDO d'ABS) sont, en effet, garantis par des assureurs spécialisés, dits « monoline ». ${ }^{21}$ 
Ces exemples montrent une grande hétérogénéité dans les solutions retenues. Contrairement à l'exemple précédent des futures, le terme français n'a pas encore été normalisé. Les journalistes ont alors été obligés de trouver des solutions ad hoc qui vont du calque à l'explicitation, ce qui montre bien la tension entre la nécessité de «faire court » et le souci d'informer le lecteur de la façon la plus complète. Dans la deuxième perspective, le dernier exemple correspond en tout point à la démarche d'un traducteur : explicitation du terme par une formulation claire, qui le replace dans son aire géographique et qui introduit également le terme source par le biais d'un marqueur d'équivalence. Cette formule permet par la suite de reprendre le terme court subprime sans l'expliciter à nouveau. Le journaliste, du fait d'autres contraintes, ne suit pas toujours cette démarche.

\section{Un traitement différent de l'information et de la langue}

Si la vérification de l'information elle-même s'impose pour le journaliste professionnel, celle de la langue de sa propre traduction est peut-être jugée moins nécessaire. À cela s'ajoute la tendance à croire que l'on comprend parfaitement une langue et qu'on est capable de tout traduire parce qu'on communique assez bien (particulièrement en anglais). Il n'est pas inutile de rappeler que la traduction est un métier qui nécessite des savoirs linguistiques et culturels étendus et approfondis ainsi que des savoir-faire acquis par un entraînement et une formation spécifiques (Lavault-Olléon, 1998; Gouadec, 2002), que n'ont pas suivis les journalistes.

Certains exemples montrent le choix d'une traduction délibérément interprétée afin de dramatiser et de forcer le trait, en privilégiant la formulation la plus sensationnelle. Comme le dénonçait Pierre Bourdieu à propos de la télévision, « les journalistes ont des 'lunettes' particulières à partir desquelles ils voient certaines choses et pas d'autres; et voient d'une certaine manière les choses qu'ils voient. Ils opèrent un sélection et une construction de ce qui est sélectionné. Le principe de sélection, c'est la recherche du spectaculaire ». Et derrière cette tendance qui s'amplifie dans certains médias, même s'il est manifeste que d'autres lui résistent avec force, il y a «la pure et simple soumission au public, c'est-à-dire au marché, à la loi de l'audimat » (Bourdieu, 1996).

Le traducteur ne se situe pas dans les rapports de force et de concurrence du champ journalistique et il a la chance de garder une relative autonomie. Face au même événement, son approche serait naturellement plus mesurée et plus neutre, plus respectueuse de la globalité du message : sans tomber dans le littéralisme, et tout en tenant compte du destinataire et de la fonction du texte, le traducteur donne des limites plus étroites à ses possibilités d'interprétation. Il répondra à la demande de son commanditaire en explicitant ou adaptant si besoin est, il reformulera, réécrira, corrigera l'original s'il est déficient (Allignol 2004 : 71), mais il résistera généralement à la déformation. Dans le cas de la traduction veille, il pourra sélectionner des passages et en omettre d'autres mais il les rendra de la manière la plus neutre possible. Le journaliste, lui, s'attachera plus à l'effet et s'octroiera plus de liberté pour représenter l'événement en fonction de ses convictions ou des besoins du média pour lequel il écrit.

Ces deux approches reflètent aussi des conceptions ou des priorités linguistiques divergentes, le traducteur se sentant plus attaché à la langue et à sa représentation. 

illustration. Les traducteurs francophones sont formés à respecter les positions des autorités linguistiques sur la défense de la langue française, évitant soigneusement les calques et n'utilisant les emprunts que s'ils sont attestés et lexicalisés. L'exemple du calque de futures montre non seulement un recours à la solution de facilité, mais aussi une certaine connivence entre des journalistes et des professionnels de la finance qui ne partagent pas le souci de préserver les langues de spécialité en français. La position officielle française est à ce titre exemplaire et a permis, même dans des domaines très anglicisés comme l'informatique, de maintenir une langue de spécialité française vivante, avec certes un taux élevé d'emprunts, mais qui fonctionne à l'écrit et à l'oral de manière authentiquement française. D'autres autorités, notamment pour des langues scandinaves comme le suédois, ont abandonné la partie en laissant l'informatique devenir un «domaine perdu» (Humbley, 2005: 37). Certains professionnels, qui évoluent dans des milieux très anglicisés comme la finance, aimeraient imposer un maximum de termes anglais dans leur domaine ${ }^{22}$, en défendant que l'uniformisation linguistique fait partie de la mondialisation et que les calques facilitent l'intercompréhension, position évidemment critiquée par les tenants du multilinguisme et de la traduction comme garant de ce dernier. utilisés sous cette forme par les professionnels d'un domaine, il faut reconnaître que les journalistes sont souvent les premiers à en informer le grand public. Ils se situent à l'avant-garde du transfert des réalités culturelles qui n'ont pas encore trouvé de désignation officielle et, comme dans le cas des subprime, se voient obligés de pallier le retard pris par les organismes normalisateurs.

Inversement, il convient de souligner que l'évolution de la langue est normale et souhaitable, que les emprunts y ont leur place pour combler des lacunes linguistiques, et que c'est souvent l'usage commun, lui-même amplifié par la presse, qui impose un terme ou une expression dans la langue. Un exemple de calque qui est entré dans la langue est l'expression " adopter un profil bas ». Elle est apparue dans les années 1980. À l'époque, lorsque nous travaillions sur les équivalences d'expressions françaises et anglaises, « keep a low profile » pouvait se traduire par « se faire tout petit » ou « se faire oublier ». Et puis il y a eu un événement : un homme politique a fait les gros titres des médias anglophones à cause de cette attitude de «low profile»; le soir même, c'est le calque jusqu'alors inconcevable, "garder un profil bas» qui est sorti dans les manchettes françaises. L'expression "prendre, adopter un profil bas » est maintenant dans le Larousse avec la définition suivante: "adopter une attitude de mesure, de modération, dans ses propos, ses projets, ses actions, généralement pour des raisons d'opportunité » (LPLI 2007) ; elle ne choque plus, semble-t-il, et elle désigne finalement une notion légèrement différente de « se faire oublier » ou « se faire tout petit ».

En consolidant et en amplifiant des usages linguistiques spontanés, les journalistes sont donc des acteurs de l'évolution de la langue. Les traducteurs se trouvent, eux aussi, couramment confrontés à des réalités nouvelles et à des terminologies inédites, qu'ils doivent traiter pour réaliser le transfert d'informations. Médiateurs entre les usages spontanés et les normes linguistiques, ils contribuent de manière raisonnée à la néologie et à l'évolution de la langue. 


\section{Conclusion}

exemples parce que nous voulions comprendre les raisons de certaines traductions inattendues et surprenantes pour des professionnels de la traduction. La traduction étant un métier non réglementé, il est courant d'épingler des traductions erronées et d'en critiquer les auteurs. Il existe certes de mauvais traducteurs, tout comme il existe de mauvais journalistes. Mais notre propos ici est davantage de souligner ce qui caractérise une formation à un métier et ce qui conduit des professionnels à travailler différemment.

31 Ainsi, dans les cours de traduction, il est habituel de travailler sur l'interprétation contextuelle des énoncés et sur la recherche d'équivalences idiomatiques non calquées (Delisle, 2003, Lavault-Olléon, 2007). Il est important aussi de sensibiliser les étudiants au fait que tout ce qui est publié dans les médias n'est pas à prendre comme référence linguistique, qu'ils doivent constamment vérifier leurs sources et retourner aux références officielles qui assurent une évolution contrôlée de la langue. Une erreur de traduction ou une formulation malheureuse peut se propager très rapidement sur Internet et donner une impression fausse de fiabilité. Ainsi une traduction calquée d'un article sur le suicide dans l'armée, publié sur CBS, mentionnait une épidémie de problèmes de santé mentale (pour l'anglais : mental health epidemic). Le problème, c'est que ce calque a été reproduit dans de nombreux articles et sites traitant du sujet des anciens combattants: les onze emplois de l'expression donnés par Google ${ }^{23}$ sont tous des citations faites à partir de cet article. On peut parler ici de véritable contamination, et le traducteur qui explore Internet pour vérifier la fiabilité d'une expression doit redoubler de vigilance.

enent, nous pensons qu'il ne serait pas inutile d'introduire des cours de traduction professionnelle dans les formations de journalistes, afin de sensibiliser les futurs journalistes aux questions de traduction, en plus des questions de langue. Certains journalistes déplorent d'ailleurs dans leur profession un « appauvrissement de la langue, qui est renforcé [...] par le comportement des rédactions des chaînes télévisées ». Ces dernières imposeraient des consignes sévères pour aider à la " communication du message », par exemple en faisant supprimer les adjectifs. Les formations auraient tendance à privilégier l'aisance d'élocution et la présentation au détriment de la maîtrise de la langue et du travail sur l'écrit (Colignon, 2007 : 43-46).

La prise en compte de ces éléments dans les formations destinées à ces deux catégories de professionnels nous apparaît d'autant plus essentielle que la révolution technologique, qui a transformé le monde en "global village», a mis à disposition de tous à la fois l'information et les moyens de la réécrire et de la modifier quasiment en direct, brouillant les sources, les contenus et les rôles. Des internautes mettent en ligne des traductions pirates (par exemple, la traduction anticipée du dernier Harry Potter ou le sous-titrage amateur des séries américaines téléchargées illégalement), d'autres s'improvisent journalistes dans des blogs qui finissent par atteindre un lectorat aussi vaste que celui d'une revue. Chacun se sent le droit, dans des forums et autres courriers de lecteurs, de critiquer les journalistes et les traducteurs et de se substituer à eux. Cette formidable liberté qu'offre Internet ne doit pas faire oublier que des formations professionnelles universitaires de haut niveau, capables de s'adapter aux évolutions en 
cours tout en associant rigueur et compétence, restent plus que jamais indispensables pour maintenir la qualité et le statut des professionnels de la communication.

\section{BIBLIOGRAPHIE}

Allignol, Claire « Le traitement des textes sources déficients, casse-tête habituel du traducteur technique », Traduire, Revue française de la traduction, Paris, SFT, n² 203, sept. 2004.

Bourdieu, Pierre, Sur la télévision, Paris, Éditions Liber, 1996.

Bourdieu, Pierre, «Journalisme et éthique », Actes du colloque fondateur du centre de recherche de l'École Supérieure de Journalisme (Lille), Les cahiers du journalisme,juin 1996, nº 1.

Colignon, Jean-Pierre, « Traducteur, journaliste, même combat », Traduire, Revue française de la traduction, Paris, SFT, $\mathrm{n}^{\circ} 212$, mars 2007.

Delisle, Jean, La Traduction raisonnée, Presses de l'université d'Ottawa, 2e édition, 2003.

Franjié, Lynne, « La traduction-veille et l'analyse médias », Traduire, Revue française de la traduction, Paris, SFT, $n^{\circ} 215$, décembre 2007.

Gouadec Daniel, Profession : traducteur, Paris, La Maison du Dictionnaire, 2002.

Humbley John, « Dictionnaires de néologismes : porte d'entrée des anglicismes ? ", Les néologies contemporaines, Paris, Société française de terminologie, 2005.

Israël, Fortunato et Lederer, Marianne (éds.) La Théorie Interprétative de la Traduction : tome I. Genèse et développement, Paris-Caen, Lettres modernes/Minard (Cahiers Champollion, $\mathrm{n}^{\circ}$ 6), 2005.

Ladmiral, Jean-René, Traduire : théorèmes pour la traduction, Paris, Gallimard 1994.

Lavault-Olléon, Élisabeth, "Traduction spécialisée : des pratiques qui se passent de théorie ? ", Traduction spécialisée : pratiques, théories, formations, Élisabeth Lavault-Olléon (éd.), Berne, Peter Lang, 2007.

Lavault-Olléon, Élisabeth « Traduction en simulation ou en professionnel : le choix du formateur ", Meta, vol. 43, n³, Les Presses de l'Université de Montréal, sept. 1998.

Lavault-Olléon, Élisabeth, « La traduction comme négociation », Enseignement de la traduction et traduction dans l'enseignement, J. Delisle et H. Lee-Jahnke (éds.), Presses de l'université d'Ottawa/ Artois Presses Université, Ottawa/Arras, 1998.

Lebtahi, Yannick et Ibert, Jérôme, « Traducteurs dans la société de l'information : Évolutions et interdépendances », Meta, Presses de l'université d'Ottawa, vol. 49, n² 2, juin 2004.

Seleskovitch Danica et Lederer, Marianne, Interpréter pour traduire Paris, Didier Erudition, 1984. Vermeer, Hans J, A Skopos Theory of Translation, Heidelberg, Textcontext Wissenschaft, 1996. 


\section{NOTES}

1. Dans le présent article, nous employons, comme le veut la langue française, le masculin singulier « traducteur " pour représenter le métier, même si celui-ci est majoritairement exercé par des traductrices.

2. Voir sur ce magazine l'article de Catherine Resche: "The Economist: discours de spécialité économique ou discours sur l'économie? » publié dans la présente revue.

3. Citons par exemple le classique manuel de Philippe Laruelle, La Version anglaise, entièrement fondé sur la traduction des encadrés de The Economist.

4. Comme il est précisé sur le site Internet du quotidien : « Nouvelobs [propose] depuis mardi 5 février, une sélection d'articles du prestigieux hebdomadaire américain Newsweek en traduction française. Les articles sont traduits par Nadia Bensmail et publiés dans leur intégralité. »

5. Voir le site.

6. Dans son article « La traduction des textes journalistiques ». M. Meertens est aussi l'auteur du Guide anglais-français de la traduction, éd 2008, Editions Chiron, Saint-Quentin-en-Yvelynes.

7. Voir le portail de l'Agence France Presse.

8. Que ces sources aient été traduites par les journalistes eux-mêmes ou reprises sans analyse critique.

9. Ces exemples sont issus d'un corpus élaboré sur une période de 6 mois entre septembre 2007 et février 2008, à partir d'articles disponibles en ligne sur les sites Internet de grands quotidiens en anglais, en espagnol et en français.

10. Les spécialistes objecteront que cela est plus vrai pour l'Espagne que pour les pays latinoaméricains où un certain formalisme demeure, notamment dans les rencontres officielles. On peut admettre dès lors que le tutoiement employé par le roi d'Espagne ait pu être perçu dans ces pays comme une marque d'arrogance rappelant la période de la colonisation.

11. Une traduction pourrait être: «Pourquoi ne te tais-tu pas?» mais nous préférons l'équivalence fonctionnelle : « Mais laisse-le parler !»

12. Une recherche sur Google donne pour «marchés des futures », entre autres : «marché des futures... mamans ».

13. Office québécois de la langue française, 2006.

14. D'après la base FranceTerme, Délégation générale à la langue française et aux langues de France : [En ligne]

15. L'express, article publié le 11 février 2008.

16. La Tribune, article publié le $1^{\mathrm{er}}$ octobre 2007.

17. Les Echos, article publié le 3 mars 2008.

18. Le Figaro, article publié le 22 janvier 2008.

19. Le Point, article publié le 9 avril 2008.

20. Le Monde, article publié le 8 septembre 2007.

21. Le Figaro, article publié le 23 janvier 2008.

22. L'Office de la langue française du Québec souligne que «la forme future est couramment utilisée dans les milieux financiers pour traduire le substantif anglais future et qu'on tente d'implanter le calque futur ... ».

23. À la date du 20 juin 2008. 


\section{RÉSUMÉS}

Cet article explore quelques points de contacts entre journalisme et traduction, principalement du point de vue du traducteur professionnel. Après avoir rappelé les différents aspects de la traduction des médias, de son usage le plus classique (à l'université) aux récentes évolutions dues à Internet, les auteurs commentent des cas où les journalistes sont amenés à traduire, et analysent quelques exemples de traductions qui leur ont paru contestables. Il en ressort un traitement différent du discours, les journalistes ayant tendance à interpréter en dramatisant, de façon à accrocher leur public ou à suivre l'orientation de leur média, contrairement aux traducteurs qui gardent une certaine neutralité. Leur position par rapport à la langue elle-même est différente, les traducteurs montrant plus de réticences, du fait de leur formation, à utiliser des calques et des emprunts que les journalistes, qui tendent à refléter les usages les plus actuels.

This paper points out some contact points between journalism and translation, mostly from the viewpoint of the professional translator. The different aspects of media translation are first described, from the most traditional (at university) to the latest evolution via Internet. The authors comment on instances when journalists have to translate texts themselves and then analyse a few examples of seemingly questionable translations. They point out that journalists have a different approach to discourse and tend to interpret and dramatize it in order to capture their readers'attention or to follow their medium's editorial line, unlike translators who remain more neutral. Their approach to language itself is also different, since translators, due to their training, seem to be more reluctant to use calques or borrow foreign words than journalists who tend to reflect trendy practices.

\section{INDEX}

Mots-clés : journalisme, traduction, traduction des médias

Keywords : journalism, media translation, translation

\section{AUTEURS}

\section{ÉLISABETH LAVAULT-OLLÉON}

Université Stendhal-Grenoble 3

ILCEA / GREMUTS

VÉRONIQUE SAURON

Université Stendhal-Grenoble 3

ILCEA / GREMUTS 